\title{
Pure SU(3) Potentials
}

\author{
Sedigheh Deldar* a

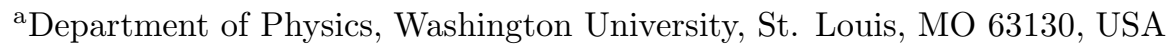

The string tensions for fundamental and a variety of representations: adjoint, 6, 10, 15-symmetric, 15antisymmetric, and 27 have been measured in pure gauge SU(3). The calculations have been done on anisotropic lattices, using an $O\left(a^{2}\right)$ tadpole improved action. A range of lattice spacings and lattice sizes has been used to control finite volume and finite lattice spacing effects. Potentials between quarks in various representations have also been calculated with the fat center vortices model. At intermediate distances, the results show approximate Casimir scaling. From my lattice calculations, no color screening has been observed so far, even for distances as large as about $2.5 \mathrm{fm}$.

One of the main goals of a non-perturbative formulation of gauge theories is to understand the phenomenon of confinement. The formation of a flux tube and the linear confinement of static quarks in the fundamental representation has been well established by pure gauge lattice QCD. The confinement of static sources in higher representations of QCD is still a question. Some numerical calculations have been done for $\mathrm{SU}(2)$ [1] and $\mathrm{SU}(3)$ [2] at non-zero temperature. At zero temperature, there have been studies for 8 (adjoint) [3], and 6 and 8 [4]. At Lattice98, I gave some preliminary results of string tensions of some higher representations at zero temperature [5]. Independent of this work, at Lattice99, G. Bali reported some results of static source potentials of some higher representations [6].

In this paper, I give results of measuring the potentials and string tensions of static sources of fundamental and some higher representations (6, $\left.8,15_{a}, 10,27,15_{s}\right)$ in pure $\mathrm{SU}(3)$. Each representation can be labeled by the ordered pair $(n, m)$, with $\mathrm{n}$ and $\mathrm{m}$ the original number of 3 and $\overline{3}$ which participated in constructing the representation. Screening is expected to occur for representations with zero triality: $8 \equiv(1,1), 10 \equiv(3,0)$, and $27 \equiv(2,2)$. (Triality is defined as $(n-m) \bmod$ 3.) For these representations, as the distance between the two adjoint sources increases, the potential energy of the flux tube rises. We expect a

\footnotetext{
*Poster presented at the 17th International Symposium,
} Lattice99, Pisa, June 29th to July 3rd, 1999. pair of gluons to pop out of vacuum when this energy is equal or greater than twice the glue-lump mass. For large distances, the static sources combine with the octet(8) charges (dynamic gluons) popped out of the vacuum and produce singlets which screen. Therefore the potential between static sources is no longer $R$ dependent. Static sources in representations with non-zero triality, $6 \equiv(2,0), 15_{a} \equiv(2,1)$ and $15_{s} \equiv(4,0)$, transform into the lowest order representation (3 and $\overline{3}$ ) by binding to the gluonic $8^{\prime} s$ which are popped out of the vacuum. As a result, the slope of the linear potentials of the representations with nonzero triality changes to the slope of the fundamental one, and a universal string tension is expected for large distances.

In this work, the potential, $V(r)$, has been found by measuring Wilson loops. At large $t$, $W(r, t) \simeq \exp [-V(r) t]$ where $W(r, t)$ is the Wilson loop as a function of $r$, the spatial separation of the quark, and the propagation time $t$. Wilson loops of higher representations have been found in terms of Wilson loops in the fundamental representation [5]. To calculate the string tensions, the potentials obtained from Wilson loops have been fitted to a linear plus Coulombic form.

The tadpole-improved tree level action of ref. [7] is used. This action has $a_{t} \neq a_{s}$, where $a_{t}, a_{s}$ are the temporal and spatial lattice spacings, respectively. For $a_{t} \ll a_{s}$ the discretization error is of order $O\left(a_{s}^{4}, a_{t}^{2}, a_{t} a_{s}^{2}\right)$. For Wilson loops, smearing followed by projection back to $\mathrm{SU}(3)$ is performed 


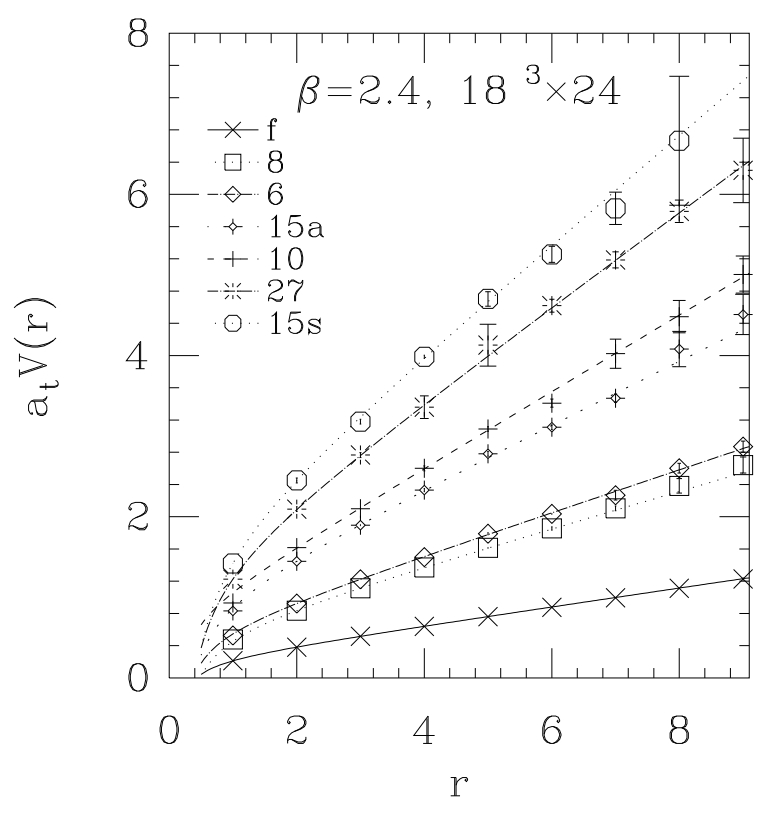

Figure 1. A typical plot of $\mathrm{V}(\mathrm{r})$ versus $\mathrm{r}$ for various representation of $\mathrm{SU}(3) . a_{s} \approx .25 \mathrm{fm}$.

on spatial links.

Measurements are done on four lattices: $10^{3} \times$ $24,8^{3} \times 24,18^{3} \times 24$, and $16^{3} \times 24$ at $\beta$ equal to $1.7,2.4,2.4$ and 3.1 , with aspect ratios of $5,3,3$, and 1.5 , respectively. The temporal lattice spacings are kept the same. Coupling constants have been chosen based on the approximate desired lattice spacing ratios: 4:2:1. Fig 1 shows a typical plot of $V(r)$ versus $r$ for various representations on the $18^{3} \times 24$ lattice at $\beta=2.4$. The string tensions are qualitatively in agreement with Casimir scaling. No color screening or change of the slope is observed for the adjoint or other representations. (Screening should start at about $1.2 \mathrm{fm}$ for the adjoint representation. 叫)

To study the scaling behavior, the potential between the static sources is found in terms of hadronic scale, $r_{0},\left(\left[r^{2} d V / d r\right]_{r=r_{0}}=1.65\right)$, where $V$ is the potential between quarks in the fundamental representation. In Fig 2, the potential between two sources in fundamental representation in terms of $r_{0}$ is plotted for different lattice measurements. Good scaling behavior is observed for the fundamental representation. The scaling gets worse for higher representations, roughly in

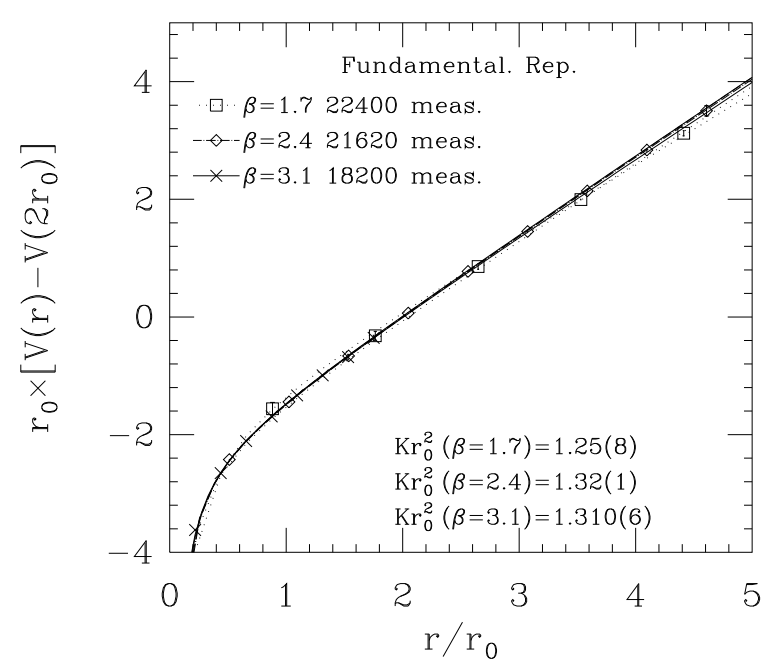

Figure 2. The static quark potential $V(R)$ in terms of hadronic scale $r_{0}$ for fundamental representation. Points are the results of three different measurements with coupling constants equal to 1.7, 2.4 and 3.1.

proportion to the string tension. Table 1 shows the best estimate for the string tension of each representation obtained by the weighted average of the four lattice measurements. The errors on the string tensions are the statistical error (from the weighted average), and the systematic error of discretization (determined by the standard deviation of the results over the 3 couplings), and the error on $r_{0}$, respectively.

I also examined the fat-center-vortices model [8] to study its prediction for the potentials. In this model, the vacuum is a condensate of vortices of finite thickness. Confinement is produced by the independent fluctuations of the vortices piercing each unit area of a Wilson loop. The average Wilson loop in $\mathrm{SU}(\mathrm{N})$, predicted by this model has the form:

$$
<W(C)>=\prod\left\{1-\sum_{n=1}^{N-1} f_{n}\left(1-\operatorname{Re}_{\mathcal{G}_{r}}\left[\vec{\alpha}_{C}^{n}(x)\right]\right)\right\},(1)
$$

where $\mathcal{G}_{r}[\vec{\alpha}]=\frac{1}{d_{r}} \operatorname{Tr} \exp [i \vec{\alpha} \cdot \vec{H}] . d_{r}$ is the dimension of representation $\mathrm{r}$, and $\left\{H_{i}\right\}$ is the subset of the generators needed to generate the center of the group. $\alpha_{C}(x)$ depends on the vortex location, and $f_{n}$ represents the probability that any given unit area is "pierced" by a vortex. I have used 
the same function for $\alpha$ as Greensite et al. used but with the appropriate normalization factor for $\mathrm{SU}(3)$. I have found $\left\{H_{i}\right\}$ for each representation in $\mathrm{SU}(3)$. Fig 3 represents the potentials between the static sources obtained by this model. For $R>40$, potentials for representations with zero triality are screened and the ones with non-zero triality closely parallel the fundamental potential. There exists a region, $R<20$, where the potential is roughly linear and qualitatively in agreement with Casimir scaling.

\section{Table 1}

Best estimate of string tensions in energy units from different coupling constants. The ratio of string tensions is proportional to the ratio of Casimir scaling of the last column.

\begin{tabular}{lccc}
\hline Rep. & $\mathrm{K}(\mathrm{GeV})$ & $\frac{K_{r}}{K_{f}}$ & $\frac{C_{r}}{C_{f}}$ \\
\hline 3 & $0.222(1)(8)(21)$ & - & - \\
8 & $0.437(2)(20)(42)$ & $1.97(1)(12)$ & 2.25 \\
6 & $0.514(5)(67)(49)$ & $2.32(3)(31)$ & 2.5 \\
$15 \mathrm{a}$ & $0.77(1)(15)(7)$ & $3.47(5)(69)$ & 4.0 \\
10 & $0.97(2)(27)(9)$ & $4.37(9)(123)$ & 4.5 \\
27 & $1.12(1)(20)(11)$ & $5.05(5)(92)$ & 6 \\
$15 \mathrm{~s}$ & $1.60(2)(52)(15)$ & $7.2(1)(24)$ & 7 \\
\hline
\end{tabular}

The numerical lattice calculations of this work shows the existence of a linear potential between static sources for fundamental and higher representations at small and intermediate distances. String tensions roughly obey Casimir scaling. No screening or change of the slope is observed for higher representations. This is in contrast to the prediction of the fat-center-vortices model. Probably Wilson loops do not couple well to screened states and couple primarily to string-like, confined states.

I thank my advisor Claude Bernard for his great support in this work. I wish to thank the MILC collaboration and specially Robert Sugar and Steven Gottlieb for computing resources.

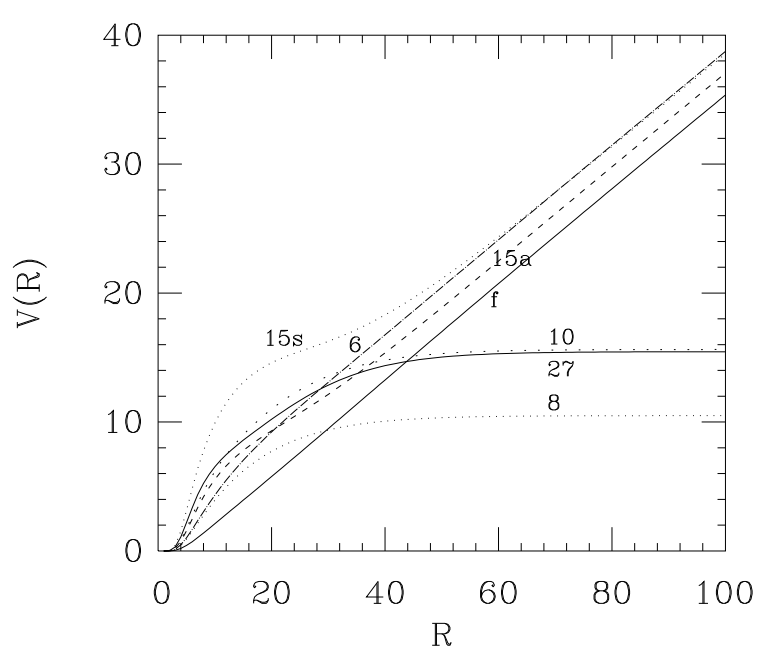

Figure 3. Potential between static sources for the range of $R \in[1,100]$. For $R>40$, potentials for representations with zero triality are screened and for the ones with non-zero triality closely parallel the fundamental potential. The fundamental representation is shown by the letter "f". The scale of $R$ and $V(R)$ are arbitrary.

\section{REFERENCES}

1. C. Bernard, Phys. Lett. 108B (1982) 431; Nucl. Phys. B219 (1983) 341; J. Ambjørn, P. Olesen, C. Peterson, Nucl. Phys. B240 (1984) 189; C. Michael, Nucl. Phys. B259 (1985) 58; G. Poulis, H. Trottier, Phys. Lett. B400 (1997) 358.

2. S. Ohta, M Fukugita, A. Ukawa, Phys. Lett. B173 (1986) 15; M.E. Faber, H. Markum, Nucl. Phys. B (Proc. Suppl.) 4 (1988) 204; M. Müller, W Beirl, M. Faber, H. Markum, Nucl. Phys. B (Proc. Suppl.) 26 (1992) 423.

3. N.A. Campbell, I.H. Jorysz, C. Michael, Phys. Lett. 167B (1986) 91.

4. C. Michael, hep-lat/9809211.

5. S. Deldar, Nucl. Phys. B (Proc. Suppl.) 73 (1999) 587.

6. G.S. Bali, hep-lat/9908021.

7. C. Morningstar, Nucl. Phys. B (Proc. Suppl.) 53 (1997) 914.

8. L. Del Debbio, M. Faber, J. Greensite, S. Olejník, Nucl. Phys. B (PS) 53 (1997) 141. 